\title{
RANDOMNESS AND DETERMINATION IN THE INTERPLAY BETWEEN THE CONTINUUM AND THE DISCRETE ${ }^{1}$
}

\author{
Francis Bailly \\ Physique, CNRS, Meudon \\ bailly@cnrs-bellevue.fr
}

\author{
Giuseppe Longo \\ LIENS, CNRS - ENS et CREA, Paris \\ http://www.di.ens.fr/users/longo
}

This paper is a conceptual analysis of the role of the mathematical continuum vs. the discrete in the understanding of randomness, as a notion with a physical meaning or origin. The presentation is "informal", as we will not write formulas; yet, we will refer to non-obvious technical results from various scientific domains. And we will propose a conceptual frame for understanding randomness (and predictability), which is essentially original, we believe. The idea we started from is that the mathematical structures, constructed for the intelligibility of physical phenomena, according to their continuous (mostly in Physics) or discrete nature (generally in Computing), may propose different understandings of Nature. In particular, as hinted in [Bailly, Longo, 2006; Longo, 2007], the causal relations, as structures of intelligibility (we "understand Nature" by them), are mathematically related to the use of the continuum or the discrete.

But... what discrete (mathematical) structures are we talking about? We believe that there is one clear mathematical definition of "discrete", that we will use in this paper: a structure is discrete when the discrete topology on it is "natural". Of course, this is not a formal definition, but in mathematics we all know what "natural" means. For example, one can endow Cantor's real line with the discrete topology, but this is not "natural" (you do not do much with it, nor you better understand the reals nor the notion of "continuous functions"); on the other hand, the integer numbers or a digital data base are naturally endowed with the discrete topology (even though one may have good reasons to work with them also under a different structuring).

In the sequel, the randomness/unpredictability issue in Quantum Mechanics is also going to be discussed. Our approach will then stress that, in the space-time of modern microphysics, in no way one may consider the discrete topology as "natural". Our work will be based exactly on quantum non-locality and non-separability results that, in our view, propose the exact opposite of an underlying discrete space-time. Indeed, the discrete topology "separates" and "localizes" the elements of a mathematical structures, this is its job. Of course, Quantum Mechanics started exactly by the discovery of a fundamental (and unexpected) discretization of light absorption or emission spectra of atoms (specifically the atom of Hydrogen). Then, a few dared to propose a discrete lower bound to measure of action, that is of the product energy $\times$ time. It is this physical dimension that bares a discrete structure. Clearly, one can then compute, by assuming the relativistic maximum for the speed of light, a Planck's length and time. But in no way space and time are thus organized in small "quantum boxes". And this is the most striking and crucial feature of Quantum Mechanics: the global and entanglement effects [Bell 1964; Bohm 1951; Aspect et al. 1982]. These effects are the opposite of a discrete, separated organization of space and time and at the core of its scientific originality. In particular, they motivate Quantum Computing (as well as our analysis of quantum randomness).

\footnotetext{
${ }^{1}$ MSCS, vol. 17, n. 2, pp. 289-307. A preliminary French version of part of this paper is the Appendix of [Bailly, Longo, 2006].
} 
As for the continuum, its role will be stressed in understanding classical determination, as mathematized in the geometry of Dynamical Systems.

As a matter of fact, the notion of randomness lies at the center of dynamic unpredictability: a deterministic system is unpredictable, precisely when it presents random evolutions. In quantum mechanics, this notion is also evoked but in a very different and intrinsic way (we will give a more precise meaning to this term). And we will attempt to examine the following issues closely: how does randomness present itself in today's natural sciences? It there a randomness specific to the various fields of Physics? What impact does this eventual differentiation/unity of the notion of physical uncertainties have on the common/scientific concept of randomness? Is it correlated to the various mathematical tools established (continuous vs. discrete, for example)?

We will not return to the terrain of specifically biological randomness (chapter 6, sect. 4.1) which remains, in our opinion, unexplored. We will firstly reflect upon classical Physics, in order to consider the problem of the meaning of randomness in the context of different theoretical frameworks. We will see that dynamical systems and Quantum Physics independently propose very important, but not identical, notions of randomness, giving rise to a different role for probabilities in their various contexts. Our distinguishing criterion will refer to the notion of "epistemicity" as being in contraposition to that of "objectivity", the difference being related to the role of the knowing subject in the construction of scientific objectivity. This is an essential role within modern Physics, particularly when it is question of probability and randomness. In short, we will first state the problem of knowing if a disordered sequence (or more generally, of a disordered state) is the effect of chaotic determinism or of pure random processes, of an epistemic or an objective nature. In the absence of very general theorems on the matter (which would contribute to a constitution of objectivity $^{2}$ ), we can develop an argumentation which will have the effect of somewhat displacing the problem all the while better highlighting some of the constraints which it implies.

We will develop the thesis that in classical Physics, randomness is of an "epistemic" nature, particularly meaning that one will always be able to interpret an apparently random sequence as stemming from a chaotic determinism or as stemming from "pure" randomness, that is analyzable in statistical terms (independently on any possible determination). Thus two different approaches are possible, according to the viewpoint one assumes. In contrast, we will call "objective" the randomness specific to quantum mechanics. Our argument will base itself upon two elements justifying this distinction. One is centered upon the role of theoretical determinism, of the "view" (constituted of the proposed theoretical framework) and of measurement. The other, stronger in a certain sense, is founded upon the properties of quantum non-separability and of continuous mathematics, which we will discuss in length.

\section{Deterministic chaos and mathematical randomness: the case of classical Physics.}

Let's therefore begin by analyzing classical randomness. To this end, we assume the idea according to which the result of a throw of dice, for example, which we legitimately consider to be random, may be interpreted as such given that the system of equations and of constraints enabling to describe it (and thus to determine it) is (very) sensitive to the initial conditions $^{3}$. In short, from the modern (post-Laplacian) point of view, even this system,

\footnotetext{
${ }^{2}$ As is the case for "mixing" random sequences (dynamics of Bernoulli), that can be demonstrated as being equivalent to (and thus interpreted as) "heads or tails" type sequences. We will return to this.

${ }^{3}$ Of course, the reverse interpretation is quite different: a dynamic system defined by its equations and
} 
paradigm of randomness, is indeed deterministic, in the sense that a sufficient number (in fact a very great number) of equations could theoretically describe all the forces at play, all of them being theoretically well-known (gravitation, various frictions...). However, this would tell us very little about its evolution: this classical system is so sensitive to the slightest variation in the boundary conditions, which are moreover very numerous, that the mathematical effort of describing the (very numerous!) equations determining it will not help us in practice, even not qualitatively. And its evolution remains unpredictable: it is that which leads us to consider it, within a classical framework, as random all the while being deterministic (chaotic).

A less familiar but simpler example may also be mentioned. Let's consider a double pendulum. We are talking about a pendulum where a second pendulum is articulated on the first, that is a weight is placed at an articulation point of the broken stick of a pendulum. This simple mechanism, perfectly determined by the two equations (it has two degrees of freedom), has a chaotic behavior: its trajectories are dense (the weights go everywhere, within the limits of their constraints), it is sensitive to the initial conditions (see [Lighthill, 1986]). Once more, the system's evolution appears random to all observers, despite the apparent simplicity of the determination. Here we have another case of epistemic randomness, which could in fact also be analyzed in terms of random sequence (by writing 0 or 1 depending on whether the smaller weight finds itself to the right or to the left after 10 oscillations, for example $)^{4}$.

A final classical example, most relevant given that it triggers all deterministic unpredictability analysis, is the "three-body problem" in their gravitational field. Poincaré demonstrated the impossibility of resolving the system of the nine Newton-Laplace equations which described its movements in either an elementary and direct manner (by using "simple" functions, let's say) or analytically (by means of convergent series). In doing so, he has enabled to analyze that which we have just done: classical determination may fail to imply predictability. With this result, he has opened the way to the integration-comprehension of classical randomness within the framework of mathematical determination: the "laws" at stake are all clarified by means of equations, however, the evolution remains unpredictable, thus, epistemically random. Modern results confirm the scientific relevance of this approach: Laskar, in his numerous articles [Laskar, 1990; 1994], has demonstrated that the solar system, our good old planetary system, is chaotic. So apart from a few differences regarding the time scales (demonstrable time of unpredictability: 1 million years for Pluto, 100 million years for Earth), it is not so different for a double pendulum, nor for a throw of dice, from the mathematical viewpoint. In the long term, we could also address it in purely statistical terms, like dice (and, for instance, bet at 2/1 that the Earth will no longer be revolving around the Sun in 500 million years).

The dynamic system which we are considering may therefore have a great number of parameters, as do dice, or a medium number of parameters, as does the solar system, or even a very small number of degrees of freedom, as does the double pendulum. On the other hand, a random representation of the statistics of the results obtained, in all of the mentioned cases, supposes a high number of parameters. We will finally note that this possibility for an alternative (chaotic determinism or randomness to be analyzed in purely statistical terms) is

presenting a chaotic behavior has an objective character associated to these equations themselves and to their intrinsic properties (it is described/given by physical objects, with their properties, their invariants, their symmetries... deduced from the equations).

${ }^{4}$ On the other hand, a simple pendulum is deterministic and, in principle, predictable. Thankfully, Galileo came across a simple pendulum. Elsewise, we would be far from understanding the law of falling bodies, in their basic simplicity... 
intimately related to the fact that local descriptions of the system are possible: those associated to the underlying equations and their initial conditions, whereas the statistical and probabilistic representations generally involve a global representation of the system (an example of this is the physical behavior of a gas, of which the thermodynamics consists precisely in taking global statistics averages - statistic mechanics - on local mechanical behaviors).

Let's finally summarize the two reasons for which we call classical randomness epistemic. First, we can choose a purely statistical mathematical approach or an analysis in terms of determination (equational). Of course, both approaches, though theoretically equivalent (and there is a sufficient amount of theorems demonstrating this equivalence), may be more or less effective or even relevant: normally, we discommend the analysis of planetary evolutions in statistical terms just as we do the analysis of dice in equational terms - each system will have its own best adapted method of analysis (in the case of a double pendulum, the difference is less clear and depends on the aims of the analysis: this pendulum may very well be used for a little family game of chance). Second, the unpredictability of a deterministic system is due to a (classical) physical principle: measurement is always an interval. That is, that even if we have a system of equations which determines a system, point by point (in Euclid's sense of points, or of real numbers à la Cantor), only God, Laplace quite soundly tells us, knows the world through (mathematical) points and can thus predict (and retrodict) its future (and passed) states. As far as we humans are concerned, our Physics uses approached measurements and does so by principle, because, in the worst (or best) of cases, there are classical thermal fluctuations which force approximation (the interval of measurement). Only discrete state machines (our digital computers, in particular when sequential and, thus, theoretically independent of physical contexts) access to exact data bases, in their discrete well separated topologies, and thus possess predictable evolutions. This was already highlighted by Turing, who views his machine as "laplacian" (see [Longo, 2007] for details and references). By this, if we consider a deterministic system somewhat sensitive to the boundary conditions (typically non-linear, even chaotic, of which a general definition uses also the notions of topological transitivity and density by periodic points, which we will not develop here, see [Devaney, 1989]), one has that unpredictability is the joint result of this sensitive dependency of the boundary conditions and the theoretical properties of classical measurement. Classical (and Relativistic, of course) theories thus simultaneously give us perfect determination (from God's viewpoint, but conceivable even by us, mathematically) and unpredictability. The key role of the mathematical continuum in these frames will be analyzed in section 3 .

In conclusion, the two following arguments which lead us to consider classical randomness as being epistemic:

1. The possible equivalence of the statistical view and equational determination;

2. The role of measurement (performed by the knowing subject).

Then, we could propose, for classical dynamics, a "Poincaré thesis":

"any classical random process is a trajectory given by a system which is, in principle, deterministic and in chaotic regime".

This thesis does not necessarily correspond to Poincaré's thinking, but to what we can say 120 years after his great theorem. It is, at most, a thesis, because it remains clearly indemonstrable (where is this list of all classical processes?), though it is falsifiable.

\section{The objectivity of quantum randomness.}


One may argue that in contrast, Quantum Physics proposes an "objective" randomness, intrinsic to the theory, conceptually and mathematically quite different from classical randomness (in [Mugur-Schachter, 2006] it is called "primordial"). This randomness is intrinsic inasmuch as it is associated to any operation of measurement, because, in Quantum Physics, a measurement only returns a probability as result. More specifically, the objective randomness of Quantum Physics is due, conjunctly (but in each of the cases quite differently evoked here):

1. To the non null value of the Planck constant $h$ (which constitutes a lower limit for the product of the possible precisions in the simultaneous measurement of two conjugated variables, namely, for the volume of the phase space, position and impulse);

2. To the process of measurement specific to Quantum Physics ("projection of the state vector" which does not depend upon the value of h, all the while being "nondetermined" and returning a value as a probability);

3. To the complex aspect of the wave function (by which what is added, complex values - superimposition principle -, is not which is measured, real numbers; the probability amplitudes do not coincide with the probabilities themselves).

Concerning the two first points, the difference with regard to classical measurement is well understood. Quantum theory is centered upon this essential role of indetermination in measurement: by the theoretical choice inherent to the approach itself, determination by points (of Euclid-Cantor, as in the mathematics of classical determination) is not always possible; such a general determination is theoretically inconceivable, even proscribed, conversely to what is presumed by the mathematics of classical deterministic systems (and which we have called the "Poincaré thesis"). The mathematics of Quantum Physics get started with Plank's h: it develops through an analysis of quantum states in terms of vectors (state vectors or, in other words, wave functions) within a space of infinite dimension (Hilbert space: the space of complex functions the squares of which are integrable). It then comes to propose a linear field where these wave functions are given in terms of complex components. Measurement, which always provides real numbers as a result, is instead associated to an essential loss of information, due to the passing from a complex variable to its absolute value. The physical relevance of the mathematical representation by complex numbers (or phase) is shown by phenomena such as quantum interference, whereas a representation as punctual particles was "classically" expected (cf. Young experiments). Similar reasons are at the basis of quantum entanglement (see below).

So here we already have a few good reasons to consider randomness as intrinsic to the theory: it stems from the measurement, the mathematics, and the evolution of the system (the wave function).

To these it would be necessary to add the intrinsic character of quantum fluctuations (in contrast to classical fluctuations related to temperature, for instance). In the measurements, this characteristic is manifested through both the residual energy of the harmonic oscillator (at the absolute zero, $0^{\circ} \mathrm{K}$ ) for instance, as well as through the resonance widths in particle theory. In short, $0^{\circ} \mathrm{K}$, in classical Physics, corresponds to the absolute absence of energy, whereas in quantum mechanics, residual energy is admitted, this playing a remarkable role (speculatively) in cosmology, in the destabilization of the "quantum void" (fundamental energetic state) during the "big-bang",

\footnotetext{
${ }^{5}$ Regarding the big-bang, one will notice (with a few word plays) that this representation resolves at the same time the enigma of the Lucretian clinamen (of which we have always wondered what could be the origin without any external influence: we find it here in the intrinsic fluctuations) as well as resolving Leibnizian perplexity (why is there something rather than nothing? ). Well, because
} 


\subsection{Separability vs. non-separability .}

Let's now present our main and possibly new argument for these analyses of randomness (classical vs. quantum). Objective (quantum) randomness appears to be deeply coupled with the properties of non-separability (the equation which describes the evolution of the system produces an entangled result for the quantons having interacted), as well as with the properties of non-locality (the measurement performed upon one of the quantons having interacted produces instantaneous "information" regarding the other's state). It is this indeed which leads to refute any local causal representation which would seek to account for specific quantum properties (technically, this refutation is ensured by the Bell inequalities [Bell 1961] which are in turn validated by the Aspect experiments [Aspect et al. 1982]). In this sense, therefore, the situation of a quantum system may always be considered as solely global, without it being possible to reduce it to a combination of local components (which appears to be indissociable from the possibility of establishing causal/deterministic evolution equations) ${ }^{6}$.

This point of view could indicate that in order to account for the situations we have just evoked, rather than resorting to the concepts of "chaotic determination" on the one hand and of "intrinsic randomness" on the other, it would be even more enlightening to recourse to the concepts of "separability" (to characterize the deterministic side) and of "nonseparability" (to characterize the intrinsically or objectively random aspect - as participant to the construction of scientific objectivity in Quantum Physics). In short, it is the possible separation of the different "objects" which participate in a classical process (each planet of an astronomical system, dice, a simple or double pendulum...) which enables its mathematical description-determination of observable evolutions. In sufficiently sensitive systems to boundary conditions, these objects may evolve in an unpredictable way, although individually always theoretically determined by the dynamic's equations. Quantum Physics, on the other hand, following non-separability (aspect of globality associated to non-locality), definitely confers to randomness a character, which is different, intrinsic, we say. Typically, if two flipping coins interact in any classically possible way, then separate while flipping in the air and fall, their analysis may be based on independent probabilities: the observation of one coin sets no limitation on the observation of the other. In contrast to this, two interacting quantons are entangled, that is the measurement of one of them sets limitations on the measurement of the other (they cannot be "separated", or are entangled). In general, thus, a set of $\mathrm{n}$ classical random events may be analized in statistical terms and it follows classical laws of probability distribution, while quantum observables may violate them, for example when they depend on entangled particles.

In the sequel we will go back to the role of trajectories in a space-time continuum as for the classical notion of determination. Now, for a quantum system, there are no hidden variables nor equations, which would determine its evolution - or the "trajectory" of a quanton

\footnotetext{
"nothing", the quantum void, is unstable all the while being submitted to these same intrinsic fluctuations: unstable (quantum) void, fluctuation and ... big bang.

${ }^{6}$ Of course, we will not address here Schrödinger's - local - equation which describes the evolution of a complex state vector and which is beyond the operation of measurement (the latter concerning another state vector involving the measurement device itself - cf., among others, decoherence theory [Zurek 1991], which corresponds, from the mathematical standpoint, to the passing from complex values to real numbers).
} 
in an underlying continuum, and this corresponds to the absence of a possible local determination? ${ }^{7}$.

In conclusion, the classical deterministic chaotic processes (dice, a double pendulum, in contrast to a simple pendulum, but even the solar system) normally also enable another description, in purely statistical terms and this follows classical probabilities. Thus, given that classical physical reality depends on this double description and on the physical (and not mathematical) nature of the limits of measurement, we have insisted on the epistemic character of classical randomness (it would depend on the approach, as is the case for the relationship between statistical mechanics and thermodynamics). On the other hand, in Quantum Physics, there is no possible double representation: any data, which would enable to access (to construct) knowledge, any measurement, is a probability, yet of a different nature from the classical one.

Of course, it is question here of an approach which would need to base itself upon somewhat general theorems in order to be further argued. It nevertheless remains that the quantum situation essentially differs from the classical situation regarding the status of the probabilities and randomness it involves and that we cannot avoid to take this situation into consideration.

\subsection{Possible objections.}

What are the objections can we formulate regarding such a point of view?

1 - Firstly, that in a way comparable (all the while being different) to the quantum situation, there could exist an intrinsic classical randomness, linked to precisely this collective effect of kinetic energy (temperature) and which would not be reducible to a dynamic system.

There are at least two possible responses to this objection. First, it is clear that the role of the initial conditions is determinant: there exists, classically - from the mathematical standpoint, a null set of measurements of the initial conditions which produces an ordered situation for a gas, for example - all speed vectors are parallel. In contrast to this, quantum non-locality and spontaneous fluctuations necessarily generate a "clinamen". Second, for a classical system, the Nernst principle ( $3^{\text {rd }}$ law of thermodynamics) states that at as the temperature approaches zero (null kinetic energy, which is conceivable for a classical system) the entropy of the system is null (complete order). Conversely, in a quantum system the indetermination relationships prohibit such a complete order even at this limit (inasmuch as one may conceive attaining it): Planck's $h$ forces a residual energy and, thus, the null kinetic energy is unconceivable ${ }^{8}$. These two counter-objections make rather implausible (or even impossible) the transferal of the quantum viewpoint to the classical frameworks (and would confirm the meaning of our "Poincaré thesis"), and viceversa: a mathematically coherent, yet limit, classical situation which has no quantum meaning".

\footnotetext{
${ }^{7}$ So-called "hidden variable" theories do not elude this type of analysis inasmuch as these variables must be considered as non-local : they cannot describe only a local dependence, on a separated quanton, but also a global dependence (in view of entanglement, see next section).

${ }^{8}$ We stress, and more we will do below, that the theoretical possibility of limit cases is crucial in classical approaches : this is the core of Cantor's continuum, a limit construction, and differential equations on it.

${ }^{9}$ An even simpler example may be given. Classically, it is theoretically conceivable to place a needle on its tip and leave it there for good (if you are very lucky): it is the epistemic nature of your shaking (approximated) hands that make it difficult. Instead, a quantum fluctuation would have it fall in a random direction, under all theoretical circumstances.
} 
2 - Then, one could more profoundly object that, despite indications to the contrary, the quantum randomness which manifests through these fluctuations is also an effect of the "viewpoint" (it is epistemic) and that the underlying agitation within an environment that one could qualify as sub-quantum (cf. Vigier, Bohm, Halbwachs, Hillion, Lochak) would enable to account for it, in as much a deterministic way as in the case of chaotic dynamic systems (Louis de Broglie's double solution theory ${ }^{10}$ or Bohm's version of hidden variables theory). This amounts to considering quantum mechanics as only "providing a statistically exact but incomplete description of physical phenomena" [de Broglie, 1961] (an incompleteness which was the perspective of the early Schrödinger or of Einstein - and more generally of E.P.R, see chapter 2).

A possible response to this objection lies in the fact that this environment itself, if one formalizes its effect, intervenes upon quantum magnitudes and their measurements in a global, non-local fashion, as we have noted earlier. Moreover, in order to justify the probabilistic properties of Quantum Physics and the presence of residual energy, of fluctuations, of virtual particles, etc. these authors referred to a sub-quantum medium or to a new concept of "ether", in principle a continuum, presenting these properties. If one cannot totally discard the possibility of such an approach, it remains that it does not respond to the heuristic principle of conceptual economy, by adding an underlying ether to phenomena (which, in the end, is not determinant). But also and foremost, from our point of view, it remains non-local and thus non-classical, within the line of that which has been demonstrated by Bell and Aspect, in view of continuous, yet non-separable, hidden variables (see chapter 2).

3 - One could still argue that this non-locality is similar to the globality presented by a classical system (the above considered gas, for instance) and therefore reinforce the representation of the effect of a statistical disorder underlying the quantum level itself.

However, we will respond that the lines of research regarding the unification of physical theories, which lead to the theories of quantum gravitation or to those of supertrings, seem to fit badly with this representation. Particularly, supersymmetries impose, on the one hand, the substitution of a non null dimensionality (strings or p-branes) to the punctual character (therefore of null magnitude) of the structures considered as elementary. On the other hand, the issues regarding the "mass of particles" open up a field of intelligibility and of objectivity which are of a different nature (Higgs fields, or supplementary "compactified" dimensions), classically unconceivable. All this is a consequence of the rupture from the punctual classical representation and the usual four-dimensional space-time, which raises these "paradoxes".

4 - Another point to take into consideration and which could nullify our distinction: the passage to the classical limit from quantum mechanics, that is, of reconstructing the classical by making $h$ tend towards 0 .

\footnotetext{
${ }^{10}$ Superimposition of a regular and extended solution of Schrödinger's equation (wave) and of a singular and much localized solution (particle) corresponding to a non-linear operator (still unknown) inserted within this equation.
} 
Now, this passing requires at least double conditions ${ }^{11}$ : on the one hand, there must be a canceling out of the Plank constant h (its value going to 0 ), and, on the other hand, it is also necessary to consider large quantum numbers (the simple canceling of $h$ does not always enable to construct the classical limit). Once these conditions are assumed, it is tempting to consider them as a way to pass to a classical limit, with its epistemic notion of randomness. In particular, it would be possible, starting from quantum non-separability (in the frame of decoherence theory, for instance, which allows to understand how the interactions with the environment - the measure apparatus for instance - destroy entanglement and nonseparability), to move away from an objective quantum randomness (if, as we hypothesize, there is a sense to this) and get to a classical epistemic doubling between random representation (purely statistical) and deterministic chaos. This would be compatible with the so called "quantum chaos", which precisely corresponds to a chaotic classical limit. Thus, many see, in the limit canceling of $h$, the possibility for a reduction to a dynamical system (thus obtaining the classical equations of mechanics) and, by resorting to large quantum numbers, the possibility for a reduction to epistemic probability. This double passing to the limit is far from being accomplished and constitutes one of the great challenges for the highly sought "unification" between the classical (and relativistic) theories and quantum frameworks.

Having attempted to respond to a few possible strong objections to our approach, there is still another aspect which militates in favor of the objective character of quantum randomness: the profound difference which exists between quantum and classical statistics. This difference, indeed, does not only stem from the non nullity of $h$, but also stems from the "observational" properties associated to classical particles in comparison to quantons and to the nature of the symmetries to which bosons and fermions respond (symmetry constraints which do not exist in the classical framework). The first are discernable whereas the latter are indiscernible, which conduces to different expressions of the statistics to which these entities obey: Fermi-Dirac for fermions (which cannot simultaneously occupy a same quantum state, and which are considered as the matter quantons, endowed with a half-full spin), BoseEinstein for bosons (which can simultaneously occupy a same quantum state, and which are considered as the interaction quantons, endowed with a full or null spin), while MaxwellBoltzmann statistics prevail for classical particles. The indiscernibility of the earlier (which is moreover related to non-separability) sends back, in our opinion, to the objective character of quantum randomness, whereas the discernability of the latter (the well isolated and "individuated" particles of which we are considering the - integral - sum of free energy) would refer to the epistemic character of classical randomness.

Moreover, we would like to add another element to this, one of a quite different nature, but one which, in our opinion, has the effect of reinforcing the objective character of quantum probabilities. As we have previously recalled, the domain of Physics where probabilities and statistics are the most present is, beyond any doubt, statistical mechanics and thermodynamics, be it at equilibrium or in the study of irreversible phenomena.

To account for these phenomena and because fluctuations (not necessarily quantum) also fill our universe at the same time as there is a certain degree of disorder, Boltzmann was lead to introduce the constant $\mathrm{k}_{\mathrm{B}}$ which bears his name. In a way, the latter measures an entropy, that is, a physical quantity generally related to averages taken from collections. The elements of these collections (a gas, for example, formed of atoms in movement submitted to random chocks) are animated by disordered movements of which the average effect of interaction is represented by the temperature T (in kelvins: K). In the simplest of cases, each

\footnotetext{
${ }^{11}$ We know that in reality the passing from the quantum to the semi-classical and to the classical is much more delicate than is presented here and that the fundamental problems are still not completely resolved.
} 
of the system's degrees of freedom is associated to an energy which is expressed under the form of: $\mathrm{k}_{\mathrm{B}} \mathrm{T} / 2$. It is to be noted that if the other physical theories (gravitational, quantum, electromagnetic,...) are likely to address isolated elements (two masses, an electron, a photon,..., in any case, elements of a limited number of degrees of freedom), on the other hand thermodynamics (domain par excellence of the relevance of the appearance and utilization of $\mathrm{k}_{\mathrm{B}}$ ) addresses situations where these elements are very numerous (high number of degrees of freedom) making it, precisely, dependent on a statistical mechanics approach ${ }^{12}$.

\subsection{Concluding remarks on quantum randomness.}

It is obviously not possible for us to provide a real conclusion : our approach is partly conjectural and to sufficiently support it there lacks general mathematical theorems (on the relationships between classical randomness and chaos). Further developments in the physical theories themselves are also needed which could nurture a vision of contemporary Physics thoroughly unified or at least sufficiently objective and discriminating regarding the processes at play in quantum interactions. Currently, the paradigm which is dominant (not necessarily meaning definitely established) rather goes in the sense of the conceptions which we have presented. But the search for a causal interpretation of Quantum Physics referring for instance to a (continuous) sub-quantum environment is not closed, even if one could consider that, since its inception, its fecundity remains somewhat limited, while progresses in other directions reveal to be quite rich. We would above all like to avoid finding ourselves in a way overdetermined by a priori views too ideological in nature which would make us want and thus defend a total determinism or, conversely, an essential indeterminism of which in any case the relevance in terms of philosophical implications remains to be demonstrated. Admitting that we nevertheless want to go beyond the operational aspect of the scientific approach in order to question the set of significations that it mobilizes (which is also among our preoccupations), it appears to us to be much more interesting to plea (and to argue) for the spatial/energetical/temporal irreducibility which is introduced by the non nullity of the $h$ constant. In fact, this irreducibility is the forebringer of considerable developments yet to be established, because it seems in our view to be correlative of these intrinsic probabilities which we have considered. These are themselves the expression, it appears, of the conditions imposed by indetermination, by the complex character of the wave function, by the noncommutativity, non-separability, etc., in short, by quantum specificities. A bit as it seems much more heuristic and fecund to plea and argue for a specificity of living phenomena, which we know to be physico-chemical in the analysis of all of its functionings, but with its mode of existence not reducing itself to this (mode of existence which conduces, in order to be understood, to the introduction of concepts as specific as those of metabolism, of normal or pathological, of living or dead, of phylogenesis or ontogenesis, even of "contingent finality", etc., cf. chapters 5 and 6 ).

Finally, it seems important to emphasize the fact that the objective character which we are assessing here is a constructed, or constituted objectivity. Its constitutive process obviously depends on the standpoint (preparation, measurements, choice of formulations, of convenient mathematical structures and principles, etc.). But once constituted, it becomes independent of the viewpoint in the sense that it recourses to abstract mathematical constants or structures which henceforth prescribe it as much as they describe it. Invariance and stability with respect to change of reference system and measure (view-point) correspond to the constituted objectivity, in science. And this brings us back to the typical play of construction

${ }^{12}$ See nevertheless $\$ 2.2$ and point 2 above for a brief commentary regarding that which Louis de Broglie has called the "thermodynamics of the isolated particle". 
principles and proof principles as well as to the richness of scientific language, between referred and referring utilization, see chapter 1 .

\section{Determination and continuous mathematics}

It is to be observed that, in the preceding sections, we have identified "random" with "unpredictable", in both Classical and Quantum Physics. In the classical case, dynamic unpredictability has provided us with the very definition of randomness, as consequence of the relationship between mathematical determination on the one hand and physical measurement on the other. In microphysics, we have highlighted that randomness is integrated to the theory itself, that it is in a way the starting point, rooted in its peculiar polarity between knowing subject and object given by means of mathematics, of the preparation of the experiments and of measurement, all conducing to an "unpredictable" result, where only probability is attained. By referring this time to the role of continuous mathematics, we will return to the difference between the two theoretical (and phenomenal) fields, which, relatively to randomness, we have separated, respectively, in terms of epistemicity and objectivity (or intrinsic for quantum theory).

When we use the classical viewpoint or tools for the analysis or the production of randomness (we observe a turbulence, throw a coin, a dice...), a preliminary analysis of the phenomenon or object is possible: we analyze the irregularities and the stabilities of a fluid, we look at the physical structure, the symmetries of a coin, of a dice.... This enables to ascribe probabilities to the process which will follow and to make physically well founded estimates regarding some elements of this process. In the case of a coin or a dice, the object's symmetries and set of physical properties enable to ascribe probabilities to the occurrence of the various possible events before they take place $(1 / 2,1 / 6$, respectively). In short, it is possible to separate the physical objects and its properties from the process, to study it before the measurement relating to the dynamic of interest to us. This is impossible in microphysics: prior to measurement, that is, before the process where randomness will be verified, it is impossible to determine the physical object with its list of properties; particularly, quantum non-separability prevents from isolating the "properties" of a quanton. In microphysics, the only form of access to the world lies in the measurement of processes. It is impossible to "look" at the photon, the electron, as we can a dice, a coin, independently of its process of production, of evolution and of measurement, where probabilities are intrinsic to observation.

Even when an information on possible measurement at the beginning of the process is given, for example, when preparing an electron for the measurement of its spin (by setting a direction, one knows a priori that it will be "up" or "down"), there is no underlying classical theory enabling us to conceive the exact theoretical determination of this spin, before and independently of measurement, as the result of a determined state or even of a trajectory. It is instead possible to conceive an analysis of the classical dynamics of a coin which, as solution to movement equations, would describe the coin's exact trajectory, the Euclidean lines of the barycenter and of a point of the edge, for example. The different "hidden variables" approaches in quantum mechanics presumed these classical theories of real underlying trajectories but, as we recalled earlier, they do not elude the non-local aspect of their specifications.

Moreover, the classical theory enables, conceptually, to go to the limit of measurement. Once again, we very well know that measurement is always an interval, in classical Physics; however, the theory of dynamical systems is given within a framework of continuous mathematics, with Euclidean points, and the trajectories are considered as a widthless lines. For this reason, as Laplace rightly stated and we already recalled, an infinite and perfect intelligence, knowing the world point by point, could predict everything, 
including throws of dice. In theory, this boundary continuous framework is, to this day, essential, because the imposing of an a priori mathematically finite limit for measurement has no classical physical sense. This then enables to conceive of the whole determination, in particular the trajectory which begins from a point; it is in this sense that the classical world is deterministic. And randomness, as unpredictability, remains epistemic: it is in the relationship between on the one hand the tool of knowledge and of determination which is mathematics, and on the other hand the object which we presume to be independent and measurable only in a humanly approximated fashion. It is this (presumed) independence which does not occur in Quantum Physics, where the object and objectivity itself are constituted by the practice of knowledge (preparation of the experiment, measurement and their mathematization, or even their principal mathematical consequence: the quantum object).

In order to better highlight the correlated role of determination and of continuous mathematics within the classical frameworks, in their autonomy relative to the physical object (a preconsitituted), let's return to an already made remark. Mathematics performs this passing to the continuous limit in many abstract constructions which are quite independent from (are at the external boundary of) the "rational" (ratios between integer magnitudes, for Greek thought). We are thinking, for instance, of the sequences of rational numbers converging towards an irrational, let's say 2: this theoretical limit produces 2, which is not a rational number. It therefore exits within a conceptual universe of actual limits (the geometric construction of 2, which so deeply troubled our colleagues in Greece, to the point of leading some of them to the brink of suicide, is the true beginning of mathematics). The understanding stemming from real numbers à la Cantor-Dedekind conferred to mathematics of continua a definitive foundation, or provided this continuum made of points beyond this world with an immense mathematical stability and conceptual invariance. It has also enabled the developments of modern Physics, where the infinite and the passages to the limit play a crucial role well beyond differential calculus (see II.2.1, chapter 2). However, it is clear that it does not have any "physical sense" if we are referring to physical measurement, although differential and algebraic calculus, in the continuous framework, are at the center of classical physico-mathematical determination. In short, classical determination is a "limit notion" and sits at the core of mathematics of continua and mathematical physics since long.

The dimensionless points, Euclid told us, are the exact departing points for trajectories, for widthless lines, he continued, determined by equations, as Newton, Laplace, and Einstein explained. It is therefore continuous mathematics which enables us to conceive, on the one hand, of theoretically perfect classical determination, and on the other, of the unpredictability of physical evolutions, somewhat sensitive to the boundary conditions, following the unavoidable imprecision of physical measurement. Because it is the very idea of a conceptually possible continuous substrate which highlights the approximation of measurement: a universe of which the spacetime is discrete, digital for instance, would be exact, because it would allow for exact measurements, digit by digit, as separable points, and an exact access to information, just as the digital machine accesses its databases.

Lets note that even in turbulence theory, the framework provided by the Navier-Stokes equations is continuous, therefore deterministic, in this limit sense, although specific to highly unpredictable phenomena (with a few ulterior important difficulties for prediction, even of the theoretical type, due to the absence, even in our day, of proof for the unicity of solutions and therefore for the unicity of the possible trajectory, once given the boundary conditions).

Yet none of this remains for Quantum Physics, as we argued: it seems impossible to pass to the threshold of possible measurement, to refer to a continuous substrate, be it purely conceptual. The theory begins with Plank's h constant, theoretical inferior boundary of measurement, indetermination intrinsic to the mathematics of quantum mechanics. There are not, in quantum space, any dimensionless points, possible departures for widthless 
trajectories: they are proscribed by the theory. In fact, there are no more trajectories whatsoever within space-time, in the classical sense: there lies the radical watershed constituted by Quantum Physics, after two thousand years of Physics of trajectories, from Aristotle to Galileo and Newton to Einstein. In this sense, randomness becomes intrinsic to the theory, participates to the construction of objectivity, itself becoming "objective".

\section{Conclusion: towards Computability}

In the previous sections we tried to understand the notion of randomness as unpredictability, in two different theoretical frames (classical dynamics and Quantum Mechanics). The epistemic nature of classical chaotic dynamics was stressed as based on

- possible alternative understandings (deterministic vs. purely statistical)

- an underlying continuum structure that allows to conceive perfect determination, in the sense of complete infinitary predictability (God, says the theory, who would know the World by Cantorian points, one by one, would be able to predict all future events).

In contrast to this, there are no alternative to measure as probability value nor (hidden) continuous variables in the (prevailing) interpretation of Quantum Mechanics, thus the "theoretically objective" nature of quantum randomness (there is no such a conceivable God in quantum theories). Moreover, the entanglement effects (non-separability and non-locality) are at the core of the prevailing interpretation of Quantum Physics and, as stressed above, they contribute the peculiar nature of quantum randomness. This interpretation is also the basis of today approaches to Quantum Computing and Cryptography (deterministic hidden variables are incompatible with current security quantum protocols).

Many computer scientists are familiar with the mathematical definition of randomness proposed by Martin-Löf, related to Kolmogorof approach and widely developed by Chaitin and others. In short, Martin-Löf used classical Computability Theory to give the notion of "passing any effective statistical test" and defined by this the so called "infinite ML-random sequences". We do not present them here formally, but we just quote one of its major consequences, which highlights the sense of the approach:

an infinite ML-random sequence has no infinite recursively enumerable subsequence.

The meaning of this strong uncomputability property should be clear: no way to predict or compute infinitely many values of the intended sequence. As a matter of fact, if you had a total recursive function that could output the date and the results of infinitely many Lotto or Bingo games, you would be very happy. Infinitely many, of course, otherwise your sequence would be "just" eventually random, which is mathematically the same, for infinite sequences.

How does this mathematical definition relate to our analysis within Physics, in terms of dynamics or processes? In the mathematical approach, based on computability, there is no reference whatsoever to an underlying physical process generating the sequence, would it be the Lotto, dies or coin tossing. By this, it is truly general and it applies as well to a quantum sequence of, say, an electron's spin-up / spin-down, interpreted as 0's and 1's (of course, this latter sequence is random or unpredictable for rather different reasons, as we said extensively : in particular, the measure itself contributes to produce the states.)

In other words, given a chaotic deterministic dynamics or a quantum phenomenon to which we can associate an infinite sequence of integers, this is ML-random. Clearly, one has to specify how to obtain a sequence from the intended process (e. g. by writing numbers or signs on dies, coins ... by quantum measurement) ${ }^{13}$. In case of a deterministic dynamics in a chaotic regime one has to wait "enough" time for unpredictability to pop out, once associated a measure and values to the process (some techniques, such as the analysis of Lyapounov

\footnotetext{
${ }^{13}$ A precise statement of this implication is still to be given (that is, characterizing the dynamics or the processes... whose chaotic behaviour engenders exactly ML-random sequances.)
} 
exponents, can given an estimate of this time: for an ago-antagonistic process modelled by the logistic functions with a minimal level of observability of, say, $10^{-15}$, one has to wait at least 50 iterations, then the kneading sequence becomes ML-random; for the evolution of the Solar system, after 1 million years the iterated analysis of the position of Pluto - still in or out of the system - is an ML-random sequence).

The use of Recursion Theory allowed Martin-Löf to set on robust grounds previous work on randomness, which was done before the invention or a sufficiently widespread use of this Theory. The notion of "passing any effective statistical test", but also its consequence in terms of inexistence of recursively enumerable subsequences, clarifies two major aspects of randomness as unpredictability.

First, it provides a frame where one can show that unpredictability is stronger than undecidability. As we already mentioned, both classical and quantum unpredictability, which we identified with physical randomness in their contexts, yield ML-randomness as a mathematical notion. In turn, the latter implies a strong form of undecidability, for a sequence: it cannot contain any recursively enumerable subsequence. It is then fair to say that unpredictability is stronger than undecidability, as non recursive enumerability, in a context where these two notions can be compared.

Second, Martin-Löf approach allows to better understand the interplay subject/object in (scientific) knowledge. There is neither randomness in Nature, nor unpredictability. The World is not random nor unpredictable, per se: this simply makes no sense. Both randomness and unpredictability pop out in the relation between the World and a knowing subject: in order to predict one needs someone to pre-dicere (latin for to say in advance). If nobody says, there is no unpredictability nor physical randomness. In Quantum Physics, there is even no "physical object" without measurement and mathematics. Now, Recursion Theory is an eminently linguistic theory: it was born as and it is a matter of algorithms, given in words within formal systems, over sequences of letters or of 0 ' and 1's. By this, it gives an important contribution to the analysis also of physical randomness, when this is defined, as we did, in terms of unpredictability. As a matter of fact, ML-randomness is based on the notion of effectiveness for a test, that this, for an activity of someone who wants to test or try to predict the evolution of a sequence. In no way the relevance of Computability in the analysis of this interplay between a knowing subject and the World proves that there is anything intrinsically computational in the world, as many claim. On the contrary, it serves only to set a limit to our linguistic (algorithmic) effort to say something about the world (to predict) and this by a negative notion of mathematical unpredictability or ML-randomness.

\section{REFERENCES}

Anandan J. "Causality, Symmetries and Quantum Mechanics" Foundations of Physics Letters, vol.15, no. 5, 415 - 438, October, 2002.

Adler R. L. Topological entropy and equivalence of dynamical systems, American Mathematical Society, 1979.

Alligood K., Sauer T., Yorke J., Chaos: an introduction to Dynamical Systems, Springer, New York, 2000.

Aspect A., Grangier P. and Roger G., "Experimental Realization of the Einstein-Podolsky-RosenBohm Gedankenexperiment : A New Violation of Bell's Inequalities”, Phys. Rev. Let. 49, p.91, 1982.

Bailly F., Longo G., Mathématiques et Sciences de la Nature. La singularité physique du vivant. Hermann, Paris, 2006. (ongoing english translation)

Bell J.S., "On the Einstein-Podolsky-Rosen Paradox", Physics 1, p.195, 1964.

Bitbol M. L'aveuglante proximité du réel, Flammarion 2000.

Bitbol M., Physique et philosophie de l'esprit, Flammarion, 2000. 
Bohm D., "The Paradox of Einstein, Rosen and Podolsky", Quantum Th. p.611, 1951.

Bohm D., La plénitude de l'univers, Paris, Le Rocher, 1987.

de Broglie L., Introduction à la nouvelle théorie des particules de M. Jean-Pierre Vigier et de ses collaborateurs, Gauthier-Villars 1961.

Devaney R. L. An Introduction to chaotic dynamical systems, Addison-Wesley, 1989.

Einstein A., Podolsky B. and Rosen N., "Can Quantum-Mechanical Description of Physical Reality be Considered complete ? " Phys. Rev. 41, P.777, 1935.

van Fraassen B. Lois et symétrie, Vrin 1994.

Laskar J. "The chaotic behaviour of the solar system", Icarus, 88: 266-291, 1990.

Laskar J. "Large scale chaos in the Solar System", Astron. Astrophys., 287, L9 L12, 1994.

Lebowitz J., "Microscopic Origins of Irreversible Macroscopic Behavior", Physica A, CCLXIII, p.516, 1999.

Lighthill J. "The recent recognized failure of predictability in Newtonian dynamics" Proc. R. Soc. Lond. A 407, 35-50, 1986.

Longo G. "Laplace, Turing and the "imitation game" impossible geometry: randomness, determinism and programs in Turing's test". In Epstein, R., Roberts, G., \& Beber, G. (Eds.). The Turing Test Sourcebook. Dordrecht, The Netherlands: Kluwer, 2007.

Mugur-Schächter M., Sur le tissage des connaissance, Hermès-Lavoisier, 2006.

Pilyugin S. Yu. Shadowing in dynamical systems, Springer, 1999.

Poincaré H. La Science et l'Hypothèse, Flammarion, Paris, 1902.

Sauer T. "Shadowing breakdown and large errors in dynamical simulations of physical systems", preprint, George Mason Univ., 2003.

Zurek W.H. "Decoherence and the Transition from the W.H Quantum to the Classical", Physics Today, p.36 October 1991.

Weyl H. Das Kontinuum, 1918a.

Weyl H. Raum, Zeit, Materie, 1918b. 